\title{
In Memory of Professor John H. Laragh
}

\author{
Massimo Volpe ${ }^{1,2}$
}

Published online: 6 May 2015

(C) Springer International Publishing Switzerland 2015

Professor John Laragh died at the age of 90 years on March 20 2015. He was graduated from Cornell Medical College and then trained in medicine at Columbia University College of Physicians and Surgeons. He was founder of the Hypertension Center at Presbyterian/Weill Cornell Medical Centers in New York in 1970, Chief of Nephrology at Columbia Physicians and Surgeons, then Chief in Cardiology of Cardiology at Weill Cornell Medical College. He was also Founder President and Chairman of the American Society of Hypertension, leading this Society to the highest levels of visibility and prominence. He was a brilliant and visionary scientist, excellent physician and intelligent mentor and leader, who inspired generations of clinicians and scientists to grow and become opinion leaders and scientific experts in their own countries.

His research was primarily focused on the pathophysiological mechanisms underlying the clinical development and progression of hypertension. He firstly demonstrated that renin, a kidney hormone, can induce and promote hypertension in humans, and then that blocking its production can reduce blood pressure levels in both experimental and clinical studies. $\mathrm{He}$ gave major contributions in identifying the key regulatory steps of the renin-angiotensin-aldosterone cascade and its clinical implications. His scientific discovery led to the development of two classes of antihypertensive drugs, namely the

Massimo Volpe

massimo.volpe@uniroma1.it

1 Division of Cardiology, Department of Clinical and Molecular Medicine, Faculty of Medicine, Sant'Andrea Hospital, University of Rome "Sapienza", Via di Grottarossa 1035-9, 00189 Rome, Italy

2 IRCCS Neuromed, Pozzilli, Italy
Angiotensin-Converting Enzyme (ACE) inhibitors and the Angiotensin II Receptor Blockers (ARBs). These drugs had a revolutionary impact on the clinical management of hypertension and hypertension-related cardiovascular diseases, substantially contributing to reducing the burden of disease and cardiovascular morbidity and mortality worldwide. On the basis of the evidence derived from pathophysiological studies and randomized clinical trials designed to test the efficacy of John Laragh's hypothesis, both ACE inhibitors and ARBs are now recommended for fist-line therapy of major cardiovascular diseases, including hypertension, diabetes, coronary artery disease, cerebrovascular disease, congestive heart failure and end-stage renal disease. His discovery was celebrated in a cover story in Time magazine in 1975.

I had the privilege of working with him several years ago at the new York Hospital Cornell University Medical College, and to collaborate with him during a life-time career. During these years, I appreciated his talent and intuition for carrying out numerous and various scientific research programs, his passion and motivation for improving the clinical management of hypertension, his attitudes and supports for ameliorating the educational mission of scientific societies and research groups, as well as his personality. My scientific and clinical formation have been significantly influenced by my experience with professor Laragh and I will never forget his intelligence, teaching and generous mentorship. The scientific figure of professor Laragh, in my opinion, has not been fully recognised by the scientific community, although his landmark scientific discoveries and achievements will remain in the history of Medicine.

Conflict of interest statement Authors have no conflict of interest to disclose. 